\title{
A Comparative Study of Active and Passive Mixers at Sub 6 GHz for Broadcasting Applications
}

\author{
G. Thirunavukkarasu' ${ }^{1}$, G. Murugesan ${ }^{2}$, S. Mithira ${ }^{3}$, \\ R.Kavimani ${ }^{4}$, M.Muthupranesh ${ }^{5}$ \\ ${ }^{1-5}$ Department of Electronics and Communication Engineering, Kongu Engineering College, Erode, \\ Indiathirueceinnovate@gmail.com ${ }^{1}$, gmece@ kongu.ac.in ${ }^{2}$, mithiras1998@gmail.com ${ }^{3}$, \\ kavimanirajesh2591999@ gmail.com ${ }^{4}$, praneshkumar4599@ gmail.com ${ }^{5}$
}

\begin{abstract}
Multiplier based mixers are frequently used in radio transceivers because of their significant mixing action. The design of down conversion mixer used in receivers is most vital as it has many challenges. The differential implementation is favored for its immunity to common mode noise, rejection of parasitic coupling and increased dynamic range. The balanced mixers perform superior than single ended mixers in terms of handling the harmonics. The balanced mixer can be constructed by using either active devices like field effect transistors (FET) or passive devices like diodes and capacitors. In this work the performance of the active mixers and passive mixers are compared for the sub 6 $\mathrm{GHz}$ band frequency for $5 \mathrm{G}$ applications like digital video broadcasting. The RF frequency of $6 \mathrm{GHz}$, local oscillator frequency of $5.9 \mathrm{GHz}$ and the IF frequency of $100 \mathrm{MHz}$ are considered. The salient features of mixers like conversion gain, noise figure, voltage standing wave ratio and isolation between ports are obtained through simulation and they are compared.
\end{abstract}

Key words: Conversion gain, double balanced mixer, isolation, overlap capacitance, $\mathrm{Sub} 6 \mathrm{GHz}$

\section{INTRODUCTION}

The mixer is an essential circuit in the front end of the communication system. It translates the information contained signal from low frequency to high frequency at transmitter and vice versa at receiver. The mixer design in very crucial in the receivers because it encounters more adverse effect from wireless channel. The receivers are classified based on the mixer as zero IF, low IF and super-heterodyne receivers. The zero IF receivers are homodyne and it does not require mixer stage. The super-heterodyne receiver and low IF receiver have mixer stage for extracting the information. The super-heterodyne receiver is usually designed with low IF in order to avoid the distortion created by inter-modulation product terms. The performance of the mixers depends on the type of circuit used to implement the mixers. Mixers are basically non-linear electronic circuits used to shift the frequency of operation and they can be designed using active devices or passive devices. Active mixers are designed using transistors and passive mixers are designed using diodes and passive components. Active mixers improve isolation between ports but they consume more power compared to passive mixer since it requires a separate dc power source. The balanced mixer topology is preferred for its ability to overcome common mode noise, parasitic coupling and enhanced dynamic range of operation. The single balanced mixer suppresses either RF or LO input but the double balanced mixer suppresses both the RF and LO input signals which has a fine operation. Double balanced mixer has increased linearity, appreciable suppression of spurious products and offers isolation between the ports. This is simulated as active mixer and passive mixer in this work. The performance of active double balanced mixer is compared with the passive mixer. This mixer comes up with an IF frequency of $100 \mathrm{MHz}$, which provides sufficient bandwidth requirement for video signal broadcasting. The following section 2 provides the literature study for motivation and references to complete this work. In section 3 the design of active double balanced mixer and passive double balanced mixers are given in to sub sections. The comparison of the performance of the active and passive mixers are given in section 4 of this paper. The conclusion is presented in section 5 and is followed by list of reference papers.

\section{RELATED WORKS}

Differential circuit based mixers are preferred in radio frequency integrated circuit design because of various advantages like noise immunity, common mode signal rejection etc. Frederick Ray I. Gomez and Maria Theresa G. De Leon have given a design of differential mixer of zero IF or direct conversion mixer [1]. They used $90 \mathrm{~nm}$ CMOS technology working at $5 \mathrm{GHz}$ for the application of WiMax receivers. They have got the conversion gain of $11.46 \mathrm{~dB}$ and noise figure of $16.53 \mathrm{~dB}$. Surbhi K.Meherwal and Prof. S. R. Diwate have given a single balanced mixer using a $90 \square$ 
hybrid coupler and to Schottky diodes and a bandpass filter working at $\mathrm{S}$ band [2]. It down converts a RF signal $2.4 \mathrm{GHz}$ to an IF of $180 \mathrm{MHz}$ by a local oscillator of $2.22 \mathrm{GHz}$. This single balanced mixer offers a conversion gain of $8.801 \mathrm{~dB}$. This mixer has been designed using strip lines. Khalid Faitah with his colleagues came up with a low power single balanced mixer design which works at $1.9 \mathrm{GHz}$ using 0.18 micrometer CMOS technology [3]. It offers the conversion gain of $7 \mathrm{~dB}$ and a low power consumption of $3.86 \mathrm{~mW}$ at $1.8 \mathrm{~V}$ supply voltage. The noise figure is $8 \mathrm{~dB}$ for this simulation. From these results they have concluded that using CMOS technology the power consumption could be reduced. Further they have given a study of using $\mathrm{SiGe}$ or GaAs for mixer design offers low noise level but the manufacturing cost is high. Lakshmi Devi R and Sreelakshmi K (2019) have given a low complexity $\mathrm{mm}$-wave Patch antenna for $5 \mathrm{G}$ applications working at $28 \mathrm{GHz}$ [4]. This design could be modified to work at sub $6 \mathrm{GHz}$ for initial deployment of 5G. This antenna provides an appreciable return loss of $-27.57 \mathrm{~dB}$ at the operating frequency.

Rahul Sharma et al., (2016) made a comparative study of different types of mixer topologies. Gilbert cell is widely used as core of the mixer because it provides high conversion gain, good port-to-port isolation and low even-order distortion [5]. Yashwini B K and Ranjani G (2019) made a study and analysis of energy conservation in wireless sensor networks. In this paper [6] the energy efficiency is analyzed. It is observed that more amount of energy is spent by communication devices and circuits which holds good for all wireless communication systems. It indicates that an efficient design of RF front end is desired. This motivates to design a low power consuming front-end circuit. Allen Gibson from his open access thesis the design and simulation of CMOS active mixer is presented. The mixer topologies in this work were designed using TSMC $0.18 \mu \mathrm{m}$ CMOS technology [7]. Four types of mixer topologies were designed and compared. In the SB Gilbert cell, the basic parameters values were ranged to better understand how the mixer reacts to changes in the three main stages: transconductance or driver stage, switching stage, and load stage. The second design used an injected method, while the third design used a double balanced. In the last design a more modern method was applied to compensate for the decrease in transistors size, as the demand of CMOS technology advances. Switched transconductance mixer offered high conversion gain of 9.246 $\mathrm{dB}$ and minimum noise figure is obtained for single balanced. Further it has the lowest power consumption of $1.084 \mathrm{~mW}$ and best $\mathrm{LO} / \mathrm{IF}$ isolation of $-37.153 \mathrm{dBm}$.

In the design guide by Steve Long a detailed design procedure of mixer is presented [8]. There would be no hope of any correspondence between simulation and measurement for the mixer characteristics that are more sensitive to model nonlinearities. Frederick Ray I. Gomez gave a study of differential mixer working as a direct conversion circuit in 90 $\mathrm{nm}$ CMOS process [9]. This mixer is working at $5 \mathrm{GHz}$ as $\mathrm{RF}$ signal frequency. This provides a conversion gain of 11.463 $\mathrm{dB}$ and noise figure of $16.529 \mathrm{~dB}$. In his design the effect of passive balun has been observed so that it reduces the conversion gain. Also, this paper suggested that active baluns are able to produce gain if it is cascaded with a double balanced mixer. However, they increase the bandwidth which is beyond the bandwidth provided by passive baluns.

Abhay Chaturvedi, Mithilesh Kumar and R. S. Meena have proposed a double balanced mixer model using 0.18 micrometer CMOS technology at $1 \mathrm{~V}$ supply voltage. The mixer used the dynamic threshold voltage MOSFET at RF and LO stages for low voltage operation [10]. The conversion gain in improved by current injection method. It took the RF frequency of around $3.4 \mathrm{GHz}$ and an IF of $264 \mathrm{MHz}$. It provides a conversion gain of $9.212 \mathrm{~dB}$ and noise figure of $6.72 \mathrm{~dB}$. This is designed for the receivers used in wireless personal area network. It used the Gilbert cell mixer as a core of the mixer because of its better isolation and high linearity.

Abbas Golmareriet with their co-authors have provided a design of low power conversion mixer for UWB applications [11]. They have used TSMC $0.18 \mu \mathrm{m}$ RF CMOS technology, working at the $3.1 \mathrm{GHz}-10.6 \mathrm{GHz}$ frequency range. The design is based on Gilbert cell architecture and current bleeding method to increase the linearity. This circuit offers a conversion gain of around $11 \mathrm{~dB}$ and return loss is below -11 $\mathrm{dB}$. The DC power consumption is found to be $8.5 \mathrm{~mW}$. These characteristics work in the wide frequency range of $3 \mathrm{GHz}$ to $11 \mathrm{GHz}$.

\section{MIXERDESIGN}

Double balanced mixers are able to provide very high levels of performance in RF or frequency mixing applications. The action of the double balanced mixer means that the input RF and local oscillator signals are "balanced out" and their level is considerably reduced at the output (see Figure1). These LO terms are cancelled at the output to zero and the down converted RF term is doubled at the output. Thus, the double balanced mixer provides large isolation between LO and IF.

The transistors M1, M2, M4 and M5 get the balanced local oscillator input. A high frequency square wave is given as LO in order to obtain increased conversion gain. Transistors M3 and M6 get the RF input which is to be down converted. The switching time of the LO transistors must be identical else the noise performance worsens. Double balancing is the basic linearization technique for active mixers. The proposed mixer is designed using NMOS technology because of its switching speed. The drain current of MOSFET device is given by (1)

$I_{D}=\mu_{n} C_{o x} \frac{W}{L}\left[\left(V_{g s}-V_{t}\right) V_{d s a t}-\frac{V_{d s a t}^{2}}{2}\right](1)$

In saturation, drain current is independent of drain voltage and square law of the gate-source voltage. The equation (1) becomes 
G. Thirunavukkarasu et al., International Journal of Emerging Trends in Engineering Research, 8(7), July 2020, 2913 - 2918

$I_{D}=\frac{\mu_{n} C_{o x}}{2} \frac{W}{L}\left(V_{g s}-V_{t}\right)^{2}$

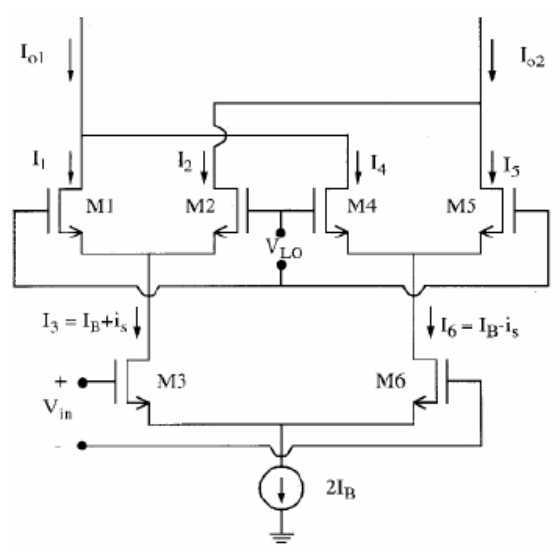

Figure 1: Double Balanced Mixer

The transconductance for this device is found to be

$g_{m}=\frac{\mu_{n} C_{o x} W}{L}\left(V_{g s}-V_{t}\right)$

This can also be represented in terms of drain current using (2) as

$g_{m}=\sqrt{2 \mu_{n} C_{o x} \frac{W}{L} I_{D}}$

From (4) it is observed that the conversion gain of the mixer is proportional to the trans conductance.

While designing the mixer it is necessary to consider the dynamic elements associated with the MOSFET device. In the reverse biased junction one can consider the standard junction capacitances as source-bulk and drain-bulk capacitance denoted by $\mathrm{C}_{\mathrm{jsb}}$ and $\mathrm{C}_{\mathrm{jdb}}$ respectively (see Figure 2). There is also small overlap between the gate-source and gate-drain; they are called as overlap capacitance of these regions. They are the important parameters of transistors used for mixer design. They will affect the high frequency performance of the mixer. The overlap capacitance is approximately given as

$C_{o v} \approx \frac{\varepsilon_{o x}}{t_{o x}} W L_{D}=0.7 C_{o x} W x_{j}$

This approximation is done by considering the amount of overlap is $2 / 3$ or $3 / 4$ times the depth of the source-drain diffusion. $\varepsilon_{o x}$ is the dielectric constant of the oxide layer of thickness $t_{o x}$ from (5). Another capacitance is gate to channel capacitance $\mathrm{C}_{\mathrm{gc}}$ given by

$C_{g c}=C_{o x} W\left(L-2 L_{D}\right)$
The capacitance between channel and bulk is given by

$$
C_{c b} \approx \frac{\varepsilon_{S i}}{x_{d}} W\left(L-2 L_{D}\right)
$$

Where $x_{d}$ is the depth of the depletion layer.

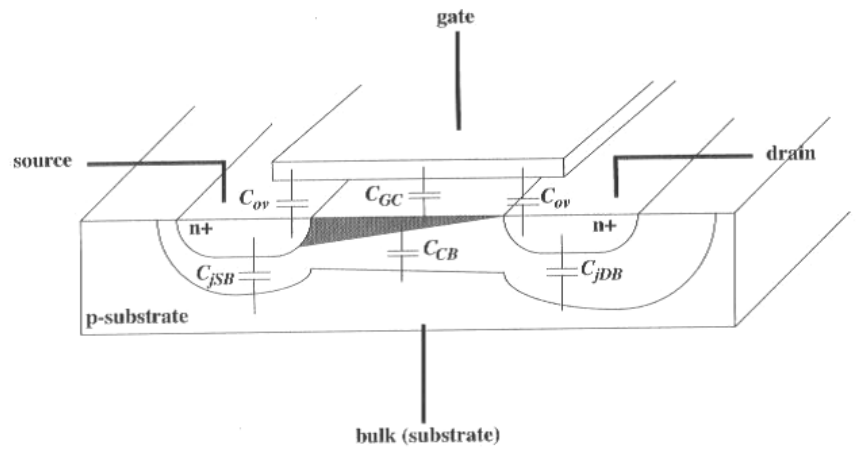

Figure 2: Capacitances in MOSFET

The operation of mixer is based on the switching between the saturation $(\mathrm{ON})$ region and the cut off (OFF) region. The MOSFETs used in the LO stage go to saturation and cut off at the rate of LO. Therefore, they must provide very good switching characteristics. The switching characteristics are influenced by these junction and stray capacitances. At saturation their gate-drain and gate-channel junction capacitance do not affect the drain current, however they influence by the overlap terms.

Another importance capacitance plays a crucial role is the drain to bulk capacitance. Table 1 shows how the capacitances change during saturation and off condition. Gate to bulk capacitance becomes zero at saturation and it has non zero value proportional to gate-channel capacitance and channel-bulk capacitance.

Table 1: MOSFET Capacitance during ON and OFF

\begin{tabular}{|l|c|c|c|}
\hline $\begin{array}{c}\text { Name of the } \\
\text { capacitance }\end{array}$ & $\begin{array}{c}\text { Notation of } \\
\text { Capacitance }\end{array}$ & $\begin{array}{c}\text { OFF } \\
\text { (cutoff) }\end{array}$ & $\begin{array}{c}\text { ON } \\
\text { (saturation) }\end{array}$ \\
\hline $\begin{array}{l}\text { Gate to drain } \\
\text { capacitance }\end{array}$ & $C_{g d}$ & $C_{o v}$ & $C_{o v}$ \\
\hline $\begin{array}{l}\text { Drain to bulk } \\
\text { capacitance }\end{array}$ & $C_{d b}$ & $C_{j d b}$ & $C_{j d b}$ \\
\hline $\begin{array}{l}\text { Gate to source } \\
\text { capacitance }\end{array}$ & $C_{g s}$ & $C_{o v}$ & $\begin{array}{l}2 C_{g c} \\
/ 3+C_{o v}\end{array}$ \\
\hline $\begin{array}{l}\text { Source to bulk } \\
\text { capacitance }\end{array}$ & $C_{j s b}$ & $C_{j s b}$ & $\begin{array}{l}C_{j s b} \\
+2 C_{c b} / 3\end{array}$ \\
\hline
\end{tabular}

\subsection{Active Double Balanced Mixer}

Active double balanced mixer (Figure 3) does simply the switching operation with perfect balancing. It requires proper impedance matching to achieve its greater balancing operation. 


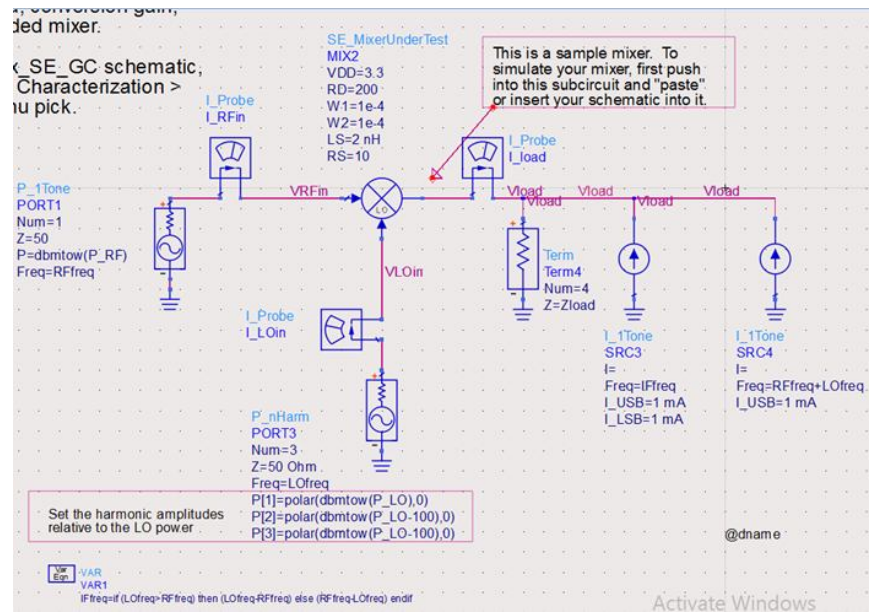

Figure 3: Mixer Functional Blocks

The impedance matching is necessary for maximum power transfer. The impendence matching is done using smith chart application in ADS EDA tool. The input section of the proposed mixer is RF side. The LO frequency is $5.9 \mathrm{GHz}$; RF is $6 \mathrm{GHz}$ and input $\mathrm{RF}$ power is $-30 \mathrm{~dB}$ are taken as the simulation parameters (see Figure 4).

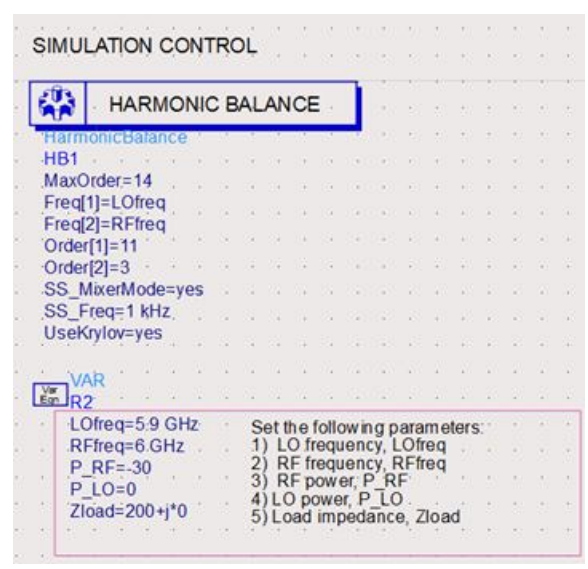

Figure 4: Simulation Specification

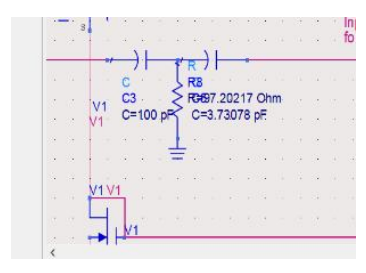

Figure 5(a): Input (RF) Matching Network

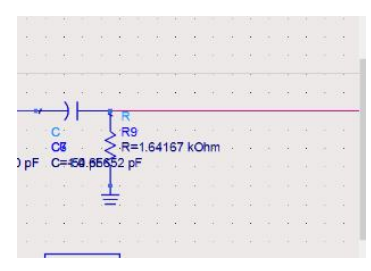

Figure 5(b: Output (IF) Matching Network
The RF input matching circuit is a T-network consisting of two capacitors of $100 \mathrm{pF}$ and $3.73078 \mathrm{pF}$. It has a shunt resistor of $97.2 \mathrm{ohms}$ (refer Figure 5a). Similarly, the output RF port is matched by an L-network consists of a capacitor of $150.65 \mathrm{pF}$ and a resistor of $1.64 \mathrm{ohms}$ (refer Figure 5b). The impedance matching results reduction of VSWR in the corresponding ports. The VSWR at RF input is 100 , after impedance matching it reduces to 1.92. The VSWR is reduced from 4 to 3.59 at the IF port.

Table 2: NMOS Simulation Specification

\begin{tabular}{|l|l|}
\hline NMOS attribute & Value \\
\hline Model & MODnmos35 \\
\hline Length & $0.1 \mu \mathrm{m}$ \\
\hline Width & $100 \mu \mathrm{m}$ \\
\hline Intrinsic length & $1.046 \mathrm{~nm}$ \\
\hline Intrinsic width & $69.025 \mathrm{~nm}$ \\
\hline Oxide layer thickness & $10 \mathrm{~nm}$ \\
\hline $\begin{array}{l}\text { Overlap capacitance } \\
\text { (gate-source, gate-drain) }\end{array}$ & $0.196 \mathrm{nF}$ \\
\hline $\begin{array}{l}\text { Effect of channel width of } \\
\text { gate due to biasing }\end{array}$ & $0.1658 \mathrm{~nm}$ \\
\hline $\begin{array}{l}\text { Effect of channel width of } \\
\text { bulk due to biasing }\end{array}$ & $5.2818 \mathrm{~nm}$ \\
\hline $\begin{array}{l}\text { Depth of source drain } \\
\text { diffusion }\end{array}$ & $150 \mathrm{~nm}$ \\
\hline \begin{tabular}{l} 
Zero bias threshold voltage \\
\hline
\end{tabular} & $0.50 \mathrm{volt}$ \\
\hline
\end{tabular}

The active double balanced mixer is designed with $0.35 \mu \mathrm{m}$ technology. The NMOS is used in the LO switching stage. The simulation specifications for NMOS and PMOS devices are given in the Table 2 and in Table 3 respectively.

\subsection{Passive Double Balanced Mixer}

Passive double balanced mixer is designed using four diodes and a center tapped transformer working at an RF frequency of $6 \mathrm{GHz}$ and an IF frequency of $100 \mathrm{MHz}$ (see Figure 6). Passive mixers are the simplest down conversion circuit used in receivers having good isolation between LO and IF also between RF and LO.

Table 3: PMOS Simulation Specification

\begin{tabular}{|l|l|}
\hline \multicolumn{1}{|c|}{ PMOS attribute } & \multicolumn{1}{c|}{ Value } \\
\hline Model & MODpmos35 \\
\hline Length & $0.1 \mu \mathrm{m}$ \\
\hline Width & $60 \mu \mathrm{m}$ \\
\hline Drain area & $0.1 \mathrm{sq} . \mu \mathrm{m}$ \\
\hline Source area & $0.1 \mathrm{sq} . \mu \mathrm{m}$ \\
\hline Perimeter of drain & $2.1 \mu \mathrm{m}$ \\
\hline Perimeter of source & $2.1 \mu \mathrm{m}$ \\
\hline
\end{tabular}


G. Thirunavukkarasu et al., International Journal of Emerging Trends in Engineering Research, 8(7), July 2020, 2913 - 2918

Normally Schottky barrier diodes are used for the diode ring. They offer a low on resistance and they also have a good high frequency response. It is found that the diode forward voltage drop for the diodes determines the optimum local oscillator drive level. RF mixers require to handle a high RF input level will need a correspondingly high $\mathrm{LO}$ input level. As a rule of thumb, the LO signal level should be a minimum of $20 \mathrm{~dB}$ higher than either the RF or IF signals.

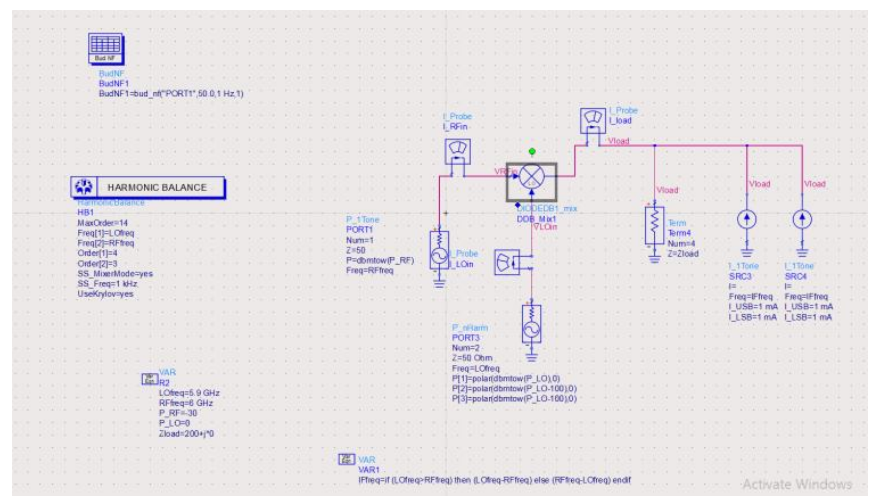

Figure 6: Passive Double Balanced Mixer - Block diagram

The operation of the double balanced mixer is relatively easy to understand (refer Figure 7). The local oscillator, LO, signal turns on first one arm (D3, D4), and then the other (D1, D2) within the diode ring.

As the points where the LO signal enters the diode ring at the junction of D1 and D4 appear as a virtual earth to the RF signal, this means that the points where the RF signal enters are alternatively connected to ground as the diodes turn on and off.The operation of the mixer means that the RF signal with alternating inverse phases is routed to the IF port according to the switching action of the local oscillator - in other words the signal at the IF port has been multiplied by the local oscillator waveform.

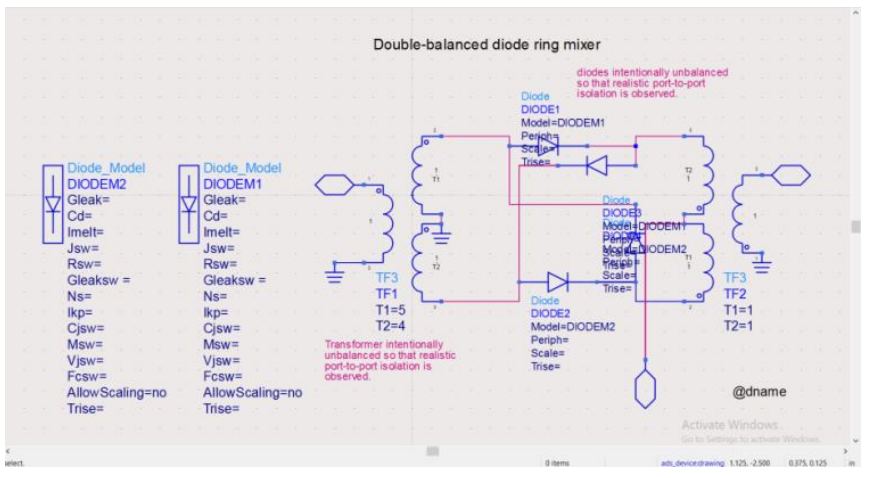

Figure 7: Double Balanced Ring Mixer

\section{ACTIVE MIXER AND PASSIVE MIXERS - A COMPARISON}

The conversion gain of down conversion mixer is the ratio of IF output power to the RF input power. It can be directly calculated by subtracting (equation 8) the RF input power from the IF output power in $\mathrm{dB}$ from the IF and RF spectrum (see Figure 8).

$G_{c}=P_{I F}-P_{R F}$
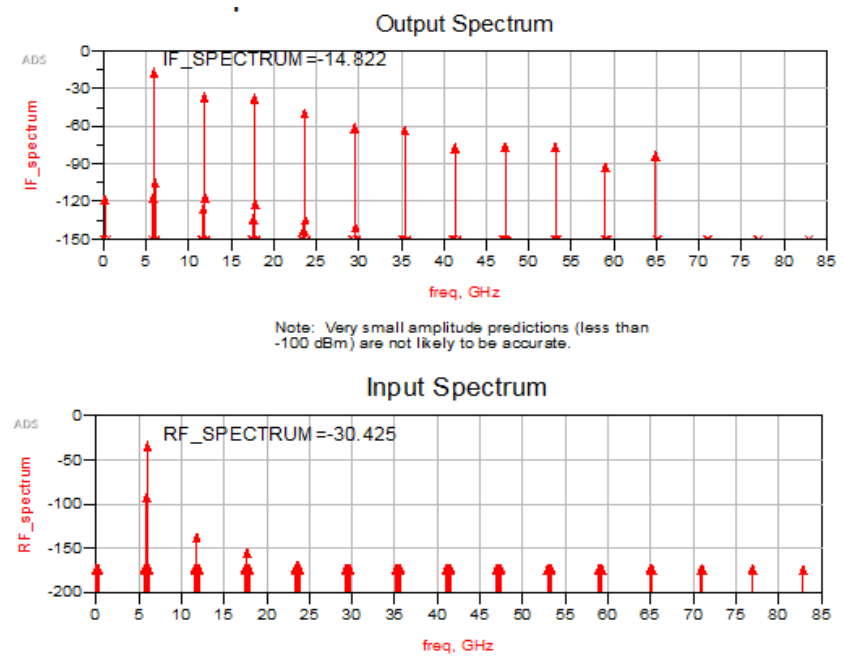

Figure 8: Input Output spectrum - Active Mixer

Conversion gain of active mixer Gca $=-14.822-(-30.425)$ $=15.603 \mathrm{~dB}$

Conversion gain of passive mixer can be calculated from its input output spectrum(see Figure 9) using equation (8).
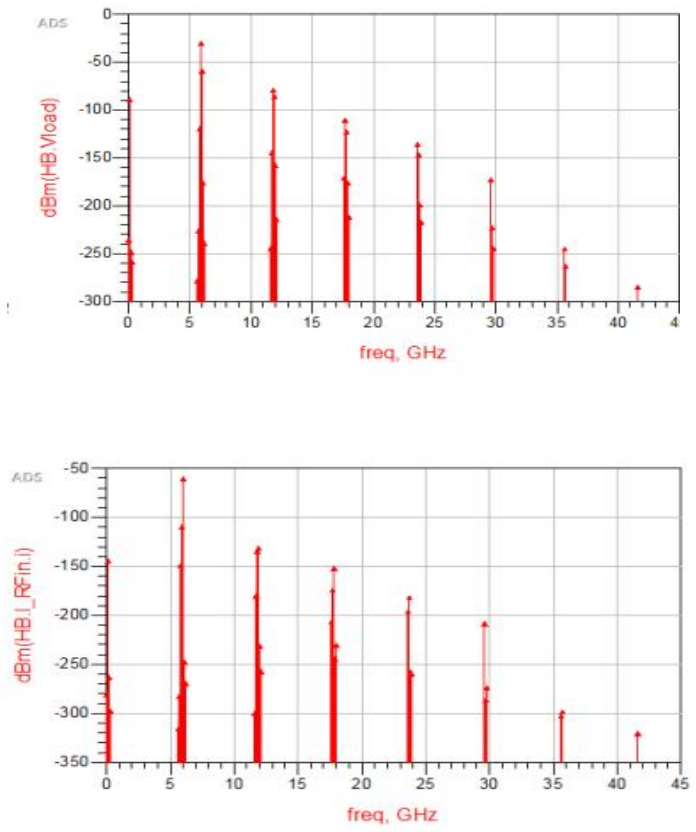

Figure 9: Input Output spectrum - Passive Mixer

Conversion gain of Passive mixer is Gcp $=-28.26-(-58.21)$ $=29.952 \mathrm{~dB}$ 
Noise figure is better in passive mixer compared with active mixer. The conversion gain is higher for passive mixer. However the RF-IF isolation is far better in the active mixer compared to passive mixer. The isolation between LO-IF is better in passive mixer. Comparison of simulation results of active mixer and passive is given in Table 4.

Table 4: Comparison of Active mixer and Passive mixer at $6 \mathrm{GHz}$

\begin{tabular}{|l|l|l|}
\hline \multicolumn{1}{|c|}{ Parameter } & \multicolumn{1}{|c|}{$\begin{array}{c}\text { Active Mixer } \\
\text { (in dB) }\end{array}$} & $\begin{array}{c}\text { Passive } \\
\text { Mixer } \\
\text { (in dB) }\end{array}$ \\
\hline Conversion gain & 15.603 & 29.952 \\
\hline Noise figure & 19.524 & 17.773 \\
\hline $\begin{array}{l}\text { Isolation between } \\
\text { RF-IF: S(1,3) }\end{array}$ & -96.347 & -28.18 \\
\hline $\begin{array}{l}\text { Isolation between } \\
\text { LO-IF: } \text { S(3,2) }\end{array}$ & -12.49 & -40.34 \\
\hline
\end{tabular}

\section{CONCLUSION}

As the double balanced mixer is preferred for its inherent fine characteristics it is most frequently employed in transceiver circuits. In this work the double balanced mixer is simulated for $6 \mathrm{GHz} \mathrm{RF}$ using the active and passive circuits. The bandwidth of $100 \mathrm{MHz}$ is sufficient for receiving the video signals. The local oscillator of $5.9 \mathrm{GHz}$ is used. From the simulation results the passive mixer has many advantages over active mixer in terms of power consumption, noise figure and conversion gain. The active mixer has the advantage over the passive mixer by its miniature size and isolation between the ports. If the application portion is concerned there are two places namely base station and mobile phones. In general low power circuits would be preferred to incubate in the mobile phones because of the limitation of battery. Hence passive mixer could be preferred to be used in the mobile devices used to receive the video broadcasting. The base station can use active mixer circuit for large power output and better isolation.

\section{REFERENCES}

1. Frederick Ray I. Gomez, Maria Theresa G. De Leon. A study of zero-IF double-balanced mixer for WiMax receivers, Heliyon(May 2019) e01741 pp. 1-6. https://doi.org/10.1016/j.heliyon.2019.e01741

2. Surbhi K.Meherwal, Prof. S. R. Diwate. Design of RF Mixer using ADS, International Research Journal of Engineering and Technology, vol.3, issue-07, July 216, pp.1823-1826.
3. K. Faitah, A. El Oualkadi, A. AitOuahman. CMOS RF down-conversion mixer for low-power wireless communications, ACM Ubiquity, Volume 9, Issue 24,June 17 - June 23, 2008, pp. 1-7. https://doi.org/10.1145/1403922.1399618

4. Lakshmi Devi R, K. Sreelakshmi, Design of Millimeter-Wave Patch Antenna for 5G Applications, International Journal of Emerging Technologies in Engineering Research, Volume 7, Issue-6, June 2019, pp. 30-33

5. Rahul Sharma, Abhay Chaturvedi and Manish Kumar. A Comparative Study of Different Types of Mixer Topologies, ICTACT Journal on Microelectronics, vol.2, issue-1, April 2016, pp. 182-187. https://doi.org/10.21917/ijme.2016.0031

6. Yashaswini B K, Ranjani G Study and Analysis of Energy Conservation in Wireless Sensor Network, International Journal of Emerging Technologies in Engineering Research, Volume 7, Issue 7, July 2019, pp. $1-5$

7. Allen Gibson, Design and Simulation of Cmos Active Mixers, (2011) Electronics Theses and Dissertations.

8. Stephen Long, RFIC Mixer design with ADS, Design Guide, $19^{\text {th }}$ April 2001.

9. Frederick Ray I. Gomez, Design and Optimization of a Direct-conversion Double-balanced Mixer for $R F$ Receiver Front end, Research Article: Progress of Electrical and Electronic Engineering, vol.1, issue-3, 2018, pp. 1-9. https://doi.org/10.18063/peee.v1i3.800

10. Abhay Chaturvedi, Mithilesh Kumar, R. S. Meena, Low Voltage Lo Noise figure Down-Conversion Mixer for Band 1 of WiMedia System, 2019 6th International Conference on Signal Processing and Integrated Networks, pp. 1137-1140. https://doi.org/10.1109/SPIN.2019.8711641

11. Mostafa Jouri, Abbas Golmakani, Design and Simulation of down-conversion CMOS Mixer for UWB applications, 2010 International Conference on Electronics Computer Telecommunications and Information Technology (ECTI-CON), pp 1-4. 